\title{
Treated depression in adolescents: predictors of outcome at 28 weeks
}

Paul Wilkinson, Bernadka Dubicka, Raphael Kelvin, Chris Roberts and lan Goodyer

\section{Background}

There is great heterogeneity of clinical presentation and outcome in paediatric depression.

\section{Aims \\ To identify which clinical and environmental risk factors at baseline and during treatment predicted major depression at 28-week follow-up in a sample of adolescents with depression.}

\section{Method}

One hundred and ninety-two British adolescents with unipolar major depression were enrolled in a randomised controlled trial (the Adolescent Depression Antidepressants and Psychotherapy Trial, ADAPT). Participants were treated for 28 weeks with routine psychosocial care and selective serotonin reuptake inhibitors (SSRIS), with half also receiving cognitive-behavioural therapy (CBT). Full clinical and demographic assessment was carried out at baseline and 28 weeks.

\section{Results}

Depression at 28 weeks was predicted by the additive effects of severity, obsessive-compulsive disorder and suicidal ideation at entry together with presence of at least one disappointing life event over the follow-up period.

\section{Conclusions}

Clinicians should assess for severity, suicidality and comorbid obsessive-compulsive disorder at presentation and should monitor closely for subsequent life events during treatment.

\section{Declaration of interest}

None.
Episodes of adolescent unipolar depression can last from weeks to many years. ${ }^{1}$ A number of depressive factors at presentation have been associated with lower treatment response and higher risk of recurrence. These include more severe depressive symptoms, suicidal ideation, melancholic features and high levels of hopelessness at presentation, ${ }^{2-7}$ together with longer duration of depression and poor social function prior to presentation. ${ }^{3}$ Non-depressive features may also influence outcome: the presence of two or more comorbid psychiatric disorders is associated with poor treatment response. ${ }^{3}$ Examination of these disorders has suggested a rather non-specific effect on treatment with no clear evidence that particular comorbid anxiety ${ }^{6}$ or behaviour disorders affect the risk of short-term persistence ${ }^{5,8}$ nor the risk of adulthood recurrence ${ }^{9}$ of major depression. The exception is the presence of concurrent obsessive-compulsive disorder, present in around $20 \%$ of adolescents with depression, which has been reported as predicting greater treatment difficulties with more prolonged episodes, independent of severity at presentation. ${ }^{5}$ Finally, psychosocial and economic factors may influence treatment response, liability for persistence or recurrence risk. For example, one naturalistic study showed that family dysfunction and friendship difficulties at presentation increase risk of persistent depression at 36 weeks. ${ }^{10}$ Undesirable life events after presentation predict persistent depression at follow-up, in particular personal disappointments in intimate social interactions with friends, ${ }^{11}$ even when baseline severity of depression is controlled for. ${ }^{12}$

Most of the above findings are reported from studies conducted in the USA using a markedly different model of healthcare availability, choice and delivery to the British National Health Service (NHS). This paper reports the influence of clinical and environmental risk factors at baseline and during treatment on whether a sample of NHS clinic-referred adolescents with depression who formed part of a larger clinical cohort involved in a randomised controlled trial ${ }^{13}$ met criteria for major depressive episode at 28-week follow-up.

\section{Method}

\section{Adolescent Depression Antidepressants and Psychotherapy Trial (ADAPT) participants}

This treatment study for adolescents with depression was undertaken through six CAMHS (child and adolescent mental health service) clinics in two main centres in England, Manchester and Cambridgeshire, from 2000 to 2004. Inclusion criteria were: ages 11-17; current full or probable (at least four depressive symptoms) DSM-IV ${ }^{14}$ major depressive disorder; and significant social impairment. Individuals were excluded if they were not suitable to be in the treatment study: immediate admission required, significant learning disability, organic cause for depression, bipolar disorder, schizophrenia, or selective serotonin reuptake inhibitors (SSRIs) contraindicated. Overall, 208 individuals entered the treatment study. The full method and clinical results are published elsewhere. ${ }^{13}$ In brief, this pragmatic effectiveness trial showed no added value for cognitivebehavioural therapy (CBT) (mean uptake of nine CBT sessions over 28 weeks) plus SSRI antidepressant over SSRI plus active routine clinical care (treatment as usual, TAU) for adolescents with moderate to severe depression by 28 weeks follow-up. Only the 192 with full major depressive disorder (at least five depressive symptoms) were eligible for the analysis reported in this paper, to facilitate generalisability of the results. Participants were assessed at baseline, 6 weeks, 12 weeks and 28 weeks by a psychology graduate or psychiatrist masked to treatment allocation. 
The study was approved and monitored by the North West Multi-Centre Research Ethics Committee and all local research ethics committees. Each participant and one adult with parental responsibility provided written informed consent.

\section{Instruments}

Kiddie Schedule for Affective Disorders and Schizophrenia, present and lifetime version (K-SADS-PL) ${ }^{15}$

The K-SADS-PL was used to measure whether DSM-IV diagnoses and individual depressive symptoms were present. Parent and adolescent data were combined to give a best estimate. Interrater reliability (kappa) for diagnosis of depression using K-SADS-PL was 0.91 on training tapes before the study and 0.71 during the study. How many of the nine DSM-IV depressive symptom clusters (e.g. depressed and/or irritable mood) were present was obtained from the K-SADS-PL.

\section{Mood and Feelings Questionnaire (MFQ) ${ }^{16}$}

The MFQ is a 33-item self-report questionnaire designed to cover the symptom areas as specified in DSM-IV for major depressive disorder. It has good test-retest reliability (Pearson's $r=0.78$ ).

\section{Children's Depression Rating Scale - revised (CDRS-R) ${ }^{17}$}

The objective severity of depressive symptoms was measured by the CDRS-R, which was designed as a paediatric version of the Hamilton Rating Scale for Depression. The CDRS-R has good criterion validity and test-retest and interrater reliability. ${ }^{17}$

\section{Health of the Nation Outcome Scales for Children and Adolescents (HONOSCA) $)^{18}$}

The HoNOSCA assesses the behaviours, impairments, symptoms and social functioning of children and adolescents with mental health problems. The higher the score, the greater the level of overall mental health problems the adolescent has. The measure is sensitive to change in mental state and psychosocial functioning over a brief (weeks and a few months) period of time.

\section{Children's Global Assessment Scale (CGAS) ${ }^{19}$}

The CGAS records the overall impression gained by the interviewer regarding the individual's current level of psychosocial functioning. The measure rates current functioning on a scale of $0-100$ on a hypothetical continuum of health $(100=$ entirely healthy) to illness ( $0=$ entirely unhealthy). Scores below 61 index children with potential mental health problems. Studies have demonstrated fair to substantial intraclass correlations of 0.59 0.90 .

\section{General Health Questionnaire-28 (GHQ-28) ${ }^{20}$}

Both parents were asked to complete the GHQ, a 28 -item selfrated questionnaire assessing mental health status over the past few weeks. It has four sub-scales: somatic symptoms (GHQ-A), anxiety and insomnia (GHQ-B), social dysregulation (GHQ-C) and severe depression (GHQ-D). For the purposes of this study, data were analysed on biological or adoptive parent(s) living at the same address as the proband. Parents also completed a demographic questionnaire about themselves and their family at the baseline assessment, which included information on household income.

\section{Recent episodic life events}

At follow-up, a life events interview was completed by the adolescent only. The methods are derived from the original studies of life events recording and measurement for adults with depression $^{21}$ and were adapted for use within adolescent populations. ${ }^{22,23}$ The methods have been adapted for use in other studies of adolescent depression. ${ }^{24}$

Recent events were included for analysis if they were initially rated by the adolescents as having a moderately to severely undesirable impact on themselves, leading to personal difficulties and lasting at least 2 weeks. A panel of individual raters also checked all life event ratings and where these events were ambiguously rated a confirmatory rating was used. Those deemed possibly not severe, low in impairment or likely to have been brought about by illness-related behaviours were excluded $(<5 \%)$. Panel raters were independent of the interviewer who collected the event information and did not know whether the adolescent had received a diagnosis at the time of their rating. Episodic events were classified into four social characteristics.

(a) Personal disappointments to the self, defined as the failure to meet prior held expectations.

(b) Physically dangerous events to self, defined as events that involved overt physical or mental risk or harm to the participant.

(c) Physically dangerous events to others, defined as events that involved overt physical or mental risk or harm to important others (family or friends).

(d) Permanent losses, defined as exit events from the participant's social field.

Validity studies by our group have demonstrated test-retest ( 2 weeks, $n=40$ ) reliability for all events (kappa $\kappa>0.85$ ) and that consensual validity with parent report is high $(\kappa=0.9) .{ }^{22,23}$ Panel raters showed good general agreement with each other (90\%) and showed high reliability with adolescents' subjective ratings of level of undesirability $(\kappa=0.8)$. Finally, there was no fall off in the retrospective reporting of events over the 12-month recall period (166 (45\%) reported one or more events, proportion reporting one or more events: 1-3 months before interview $16 \%$, 4-6 months $15 \%, 7-12$ months $14 \%$ ).

\section{Statistical analyses}

End-point depression was defined as having a diagnosis of DSM-IV major depressive episode at final 28-week assessment. All other participants (in partial or full remission) were classified as not having depression. Our data did not allow us to distinguish between individuals with depression throughout the follow-up period and those who remitted then relapsed.

Kiddie Schedule for Affective Disorders and Schizophrenia (present and lifetime version) threshold (3) levels were used to define a symptom as being present. The K-SADS-PL 'definite' levels were used to define comorbid disorders as being present. The DSM-IV definition of 'melancholia' was used (anhedonia or lack of mood reactivity; at least three of: distinct quality of depressed mood, depression worse in morning, terminal insomnia, psychomotor disturbance, anorexia or weight loss, excess guilt). Number of depressive symptom clusters refers to the nine symptom clusters utilised in the diagnosis of depression in DSM-IV. Suicidality referred to a threshold score in at least one of: recurrent thoughts of death, suicidal ideation or suicidal acts. Number of comorbid diagnoses was number out of: behaviour disorder (conduct disorder or oppositional defiant disorder); anxiety disorder (agoraphobia, panic disorder, separation anxiety 
disorder, social phobia and/or simple phobia); obsessive-compulsive disorder; attention-deficit hyperactivity disorder and eating disorder (anorexia nervosa or bulimia nervosa).

Participants with and without end-point depression were compared on demographic and clinical variables. As severity of depression is a well-proven predictor of persistent depression, a high index of suspicion was maintained that any findings may be because of severity being a confounding variable. Therefore, any individual variable predicting end-point depression on univariate analysis was entered into a multiple regression with the clinical assessor-rated severity measure, the CDRS-R, to test whether this association was independent. As it is possible that treatment centre (Manchester or Cambridgeshire) or treatment group may affect outcome, if initial analyses suggested significant results, separate confirmatory regression analyses were performed, each with one of these variables added. As it is possible that there are different effects of variables between genders and across ages, one of these variables, the putative prognostic variable and their interaction were entered into logistic regressions, with end-point depression as the outcome variable. If there were significant interaction terms, analyses were split by gender and/or age.

Variables found to be significantly associated with end-point depression were entered into a receiver operating curve analysis (ROC4), ${ }^{25}$ which identifies which variable, at which cut-point, predicts outcome most efficiently; the groups identified in each round of the analysis are then re-tested to see which variable best predicts outcome within that group, until groups are too small for significant predictors to be found $(P>0.01)$. A kappa value of 0.5 (equal preference given to sensitivity and specificity) was used.

Finally, those with and without end-point depression were compared on all clinical risk factors found to be significantly associated with end-point depression both at baseline and at 28 weeks to demonstrate whether these factors improved over treatment.

The chi-squared statistic was used to compare proportions on participants in different groups; Fisher's exact test was used if cell counts were low. If predictor data was ordinal (number of life events), chi-squared test of trend was used. Pairs of continuous variables were compared using student's $t$-test if data was normally distributed; if not, a $t$-test was carried out on log-transformed data or the Mann-Whitney test was used. For paired data (at 0 and 28 weeks), Wilcoxon signed ranks test was performed on continuous data and McNemar test was performed on dichotomous data. This was an exploratory analysis of data collected for a different purpose. A probability of $P<0.05$ (two-tailed) was therefore accepted as statistically significant in this hypothesis-generating study. Signal detection analysis was carried out using ROC4 'signal detection methods and prediction of treatment response', version 4.19. ${ }^{26}$ Other data were analysed using SPSS version 12.0 for Windows.

\section{Results}

Data on depression diagnosis at final assessment were available on 177/192 participants (92\%). Participants for whom follow-up data were available were compared with those for whom there was no follow-up data. Those with follow-up data were significantly older (mean 14.3 years $($ s.d.=1.25) v. 13.7 (s.d.=1.05), Mann-Whitney $Z=2.09, P=0.036)$. There were no significant differences in baseline CDRS-R, MFQ, age or duration of depression (all $P>0.2$ ).

Of the 177 participants available for analysis, $41(23 \%)$ had a diagnosis of major depressive disorder at 28-week follow-up, with the remaining $136(77 \%)$ in full or partial remisison. Treatment group (SSRI + TAU $v$. SSRI + TAU + CBT) had no effect on risk of being in the persistent depression group (SSRI + TAU: 18/84 $(21 \%) \quad$ v. SSRI + TAU + CBT: $\left.23 / 93 \quad(25 \%), \quad \chi^{2}=0.3, \quad P=0.7\right)$. Clinical site had no effect on this risk (Manchester: 30/121 (25\%); Cambridgeshire: $\left.11 / 56(20 \%), \chi^{2}=0.6, P=0.6\right)$.

\section{Clinical data}

Baseline demographic and clinical rating scale comparisons of participants with and without end-point depression are shown in Table 1. Greater observer (CDRS-R) and self-rated (MFQ) severity of depression, greater social impairment (CGAS), greater total mental health problems (HoNOSCA), a greater number of depression symptom clusters at baseline and greater number of comorbid psychiatric disorders were all associated with depression at 28 weeks (all $P=0.02$ ). Gender, age and duration of depression were not significantly associated with end-point depression at 28 weeks (all $P>0.4$ ).

Observer-rated severity of depressive symptoms (CDRS-R) was the variable most strongly associated with end-point depression (effect size, standardised mean difference $=0.75$ ). Increasing number of comorbid disorders $(\mathrm{OR}=1.7,95 \% \mathrm{CI}$ 1.1-2.6, Wald $=5.0, P=0.025)$ but not the number of depressive symptoms $(\mathrm{OR}=1.1,95 \%$ CI $0.8-1.5$, Wald $=0.2, \quad P=0.6)$ also contributed independently of severity. Number of comorbid disorders and CDRS were both still significantly associated with end-point depression when each was entered into logistic regressions with site and treatment group $(P=0.025)$. Age and

Table 1 Baseline demographic and illness severity for participants with and without depression at 28-week follow-up

\begin{tabular}{|c|c|c|c|c|}
\hline & No end-point depression & End-point depression & Statistic & $P$ \\
\hline Gender, $n(\%)$ & & & $\chi^{2}=0.0$ & 1.0 \\
\hline Male & $36(26)$ & $11(27)$ & & \\
\hline Female & $100(74)$ & $30(73)$ & & \\
\hline Age, years: mean (s.d.) & $14.3(1.3)$ & $14.4(1.2)$ & $Z=0.2^{\mathrm{a}}$ & 0.9 \\
\hline Duration, weeks: mean (s.d.) & $67.2(88.6)$ & $74.8(73.0)$ & $t=0.8^{b}$ & 0.4 \\
\hline Children's Depression Rating Scale - Revised, mean (s.d.) & $58.5(8.8)$ & $65.6(9.7)$ & $t=4.3$ & $<0.001$ \\
\hline Mood and Feelings Questionnaire, mean (s.d.) & $38.0(11.3)$ & $45.2(10.6)$ & $Z=3.5^{\mathrm{a}}$ & $<0.001$ \\
\hline Health of the Nation Outcome Scales for Children and Adolescents, mean (s.d.) & $25.1(5.5)$ & $27.5(5.8)$ & $t=2.4$ & 0.016 \\
\hline Children's Global Assessment Scale, mean (s.d.) & $41.4(5.7)$ & $37.4(8.2)$ & $Z=3.3^{\mathrm{a}}$ & 0.001 \\
\hline Number of symptoms, ${ }^{c}$ mean (s.d.) & $6.5(1.2)$ & $7.2(1.3)$ & $Z=2.8^{\mathrm{a}}$ & 0.005 \\
\hline Number of comorbid psychiatric diagnoses, ${ }^{\mathrm{d}}$ mean (s.d.) & $1.1(0.8)$ & $1.5(1.0)$ & $t=2.7$ & 0.008 \\
\hline $\begin{array}{l}\text { a. Mann-Whitney test. } \\
\text { b. Log transformed data. } \\
\text { c. Maximum of nine. } \\
\text { d. Maximum of five. }\end{array}$ & & & & \\
\hline
\end{tabular}




\begin{tabular}{|c|c|c|c|c|}
\hline Depressive symptom & With depression if symptom absent, $n / N(\%)$ & With depression if symptom present, $n / N(\%)$ & $\chi^{2}$ & $P$ \\
\hline Depressed mood & $5 / 18(28)$ & $36 / 159(23)$ & 0.2 & 0.6 \\
\hline Irritable mood & 16/71 (23) & 25/106 (24) & 0.0 & 1.0 \\
\hline Anhedonia & 14/82 (17) & 27/95 (28) & 3.2 & 0.1 \\
\hline Reduced appetite & 19/80 (24) & 22/97 (23) & 0.0 & 1.0 \\
\hline Increased appetite & $33 / 140(24)$ & 7/36 (19) & 0.3 & 0.7 \\
\hline Insomnia & 6/4249 (14) & $35 / 132(27)$ & 2.6 & 0.1 \\
\hline Hypersomnia & $32 / 136(24)$ & $8 / 40(20)$ & 0.2 & 0.7 \\
\hline Psychomotor agitation & 29/126 (23) & 12/51 (24) & 0.0 & 1.0 \\
\hline Psychomotor retardation & $30 / 118(25)$ & 11/59 (19) & 1.0 & 0.4 \\
\hline Fatigue & 12/50 (24) & 29/127 (23) & 0.0 & 1.0 \\
\hline Worthlessness & 12/57 (21) & 29/119 (24) & 0.1 & 0.7 \\
\hline Excessive guilt & 20/111 (18) & 21/65 (33) & 4.7 & 0.042 \\
\hline Poor concentration & 3/23 (13) & 38/154 (25) & 1.5 & 0.3 \\
\hline Indecisiveness & 22/95 (23) & 19/81 (23) & 0.0 & 1.0 \\
\hline Suicidality & $7 / 72(10)$ & $34 / 105$ (32) & 12.3 & $<0.001$ \\
\hline Hopelessness & 20/116 (17) & 21/61 (34) & 6.6 & 0.014 \\
\hline Melancholia & 30/134 (22) & $11 / 43(26)$ & 0.2 & 0.7 \\
\hline
\end{tabular}

\begin{tabular}{|c|c|c|c|c|}
\hline Comorbid psychiatric diagnosis & With depression if diagnosis absent, $n / N(\%)$ & With depression if diagnosis present, $n / N(\%)$ & $\chi^{2}$ & $P$ \\
\hline Anxiety disorder & 9/64 (14) & $31 / 112(28)$ & 4.3 & 0.041 \\
\hline Simple phobia & 26/141 (18) & $14 / 35(40)$ & 7.4 & 0.006 \\
\hline Panic disorder & 24/125 (19) & 16/51 (31) & 3.1 & 0.08 \\
\hline Separation anxiety disorder & 33/155 (21) & 7/21 (33) & 1.5 & 0.3 \\
\hline Social phobia & $22 / 111(20)$ & 18/65 (28) & 1.4 & 0.3 \\
\hline Agoraphobia & 27/122 (22) & $13 / 54(24)$ & 0.1 & 0.9 \\
\hline Obsessive-compulsive disorder & 20/126 (16) & 19/49 (39) & 10.7 & 0.002 \\
\hline Behaviour disorder & $33 / 145(23)$ & $7 / 30(23)$ & 0.0 & 1.0 \\
\hline $\begin{array}{l}\text { Attention-deficit hyperactivity } \\
\text { disorder (combined type) }\end{array}$ & 39/165 (24) & 1/9 (11) & $\begin{array}{c}\text { Fisher's } \\
\text { exact }\end{array}$ & 0.7 \\
\hline Eating disorder & $37 / 171(22)$ & $3 / 5(60)$ & $\begin{array}{c}\text { Fisher's } \\
\text { exact }\end{array}$ & 0.08 \\
\hline
\end{tabular}

gender did not moderate the effect of CDRS nor number of comorbid disorders on end-point depression (interaction terms, $P>0.1$.

Baseline comorbid psychiatric diagnoses and depressive symptoms are shown in Tables 2 and 3. The only comorbid psychiatric disorders at entry significantly associated with endpoint depression were obsessive-compulsive disorder (relative risk $\mathrm{RR}=2.4, P=0.002)$, any anxiety disorder $(\mathrm{RR}=2.0, P=0.04)$ and simple phobia $(\mathrm{RR}=2.2, P=0.006)$. The only depressive symptoms significantly associated with end-point depression at 28 weeks were higher suicidality $(\mathrm{RR}=3.3, P<0.001)$, greater hopelessness $(\mathrm{RR}=2.0, P=0.014)$ and excess guilt $(\mathrm{RR}=1.8, P=0.042)$. DSM-IV melancholia was not significantly associated with end-point depression $(P=0.7)$.

Obsessive-compulsive disorder $(\mathrm{OR}=2.8,95 \%$ CI 1.3-6.1, Wald $=11.5, P=0.010)$ and suicidality $(\mathrm{OR}=3.0,95 \%$ CI $1.2-7.5$, Wald, $P=0.021)$, but not anxiety disorder $(P=0.3)$, simple phobia ( $P=0.051)$, hopelessness $(P=0.4)$ or guilt $(P=0.15)$, were independently associated with end-point depression, independently of severity (CDRS-R score remained significant in all above regressions (all $P=0.002)$ ). Both obsessive-compulsive disorder and suicidality remained significantly associated with end-point depression when controlling for each site and treatment group (all $P=0.001$ ).

To test whether any of these risk factors of CDRS, obsessivecompulsive disorder and suicidality moderated the effects of each other on risk of end-point depression, separate logistic regressions were carried out with two of these risk factors and their interaction term as covariates for all possible pairs. In all cases, interaction terms were non-significant $(P>0.15)$. Gender and age did not significantly moderate the effects of suicidality or obsessivecompulsive disorder on end-point depression (interaction terms, all $P>0.15)$.

\section{Environment data}

Of 169 participants for whom data was available, 55 (32\%) had at least one unpleasant life event by 28 weeks follow-up. Table 4 shows that a greater total number of unpleasant life events was associated with increased risk of end-point depression $(P=0.004)$. In particular, number of life events involving disappointments $(P=0.001)$ and danger to self $(P=0.043)$ were associated with increased risk of end-point depression. To meet statistical assumptions, each life event category was dichotomised to no or at least one life event, and each entered into a separate logistic regression with baseline CDRS-R, site and treatment group as covariates. Presence of at least one disappointing life event $(\mathrm{OR}=3.8,95 \%$ CI 1.4-9.9, Wald=7.1, $P=0.008)$ or loss by death life event $(\mathrm{OR}=3.5,95 \%$ CI $1.03-11.8$, Wald $=4.0$, $P=0.046)$ were each associated with risk of end-point depression, controlling for baseline severity of depression. Presence of at least one of any type of life event $(\mathrm{OR}=1.4, P=0.4)$ was not significantly independently associated with end-point depression. Gender 
Table 4 Proportion of participants with end-point depression depending on number of 'quite' or 'very' unpleasant life events over the previous 28 weeks

\begin{tabular}{|c|c|c|c|c|c|c|}
\hline \multirow[b]{2}{*}{ Type of life event } & \multicolumn{5}{|c|}{ Life events, $n / N(\%)^{a}$} & \multirow[b]{2}{*}{$\chi^{2}$-test of trend $P$} \\
\hline & 0 & 1 & 2 & 3 & 4 & \\
\hline Disappointments & 27/146 (18) & $5 / 14(36)$ & $5 / 8(63)$ & $1 / 1(100)$ & & 0.001 \\
\hline Loss by death & $32 / 155(21)$ & 6/11 (55) & $0 / 3(0)$ & & & 0.3 \\
\hline Loss of pet & $36 / 156(23)$ & 2/11 (18) & $0 / 1(0)$ & & & 0.8 \\
\hline Loss by moving & $37 / 167(22)$ & $0 / 1(0)$ & $1 / 1(100)$ & & & 0.2 \\
\hline Danger to self & $35 / 163(23)$ & $1 / 4(25)$ & $2 / 2(100)$ & & & 0.043 \\
\hline Danger to others & $36 / 153(24)$ & 2/13 (15) & $0 / 3(0)$ & & & 0.3 \\
\hline Total life events & 23/114 (20) & $2 / 25(8)$ & $7 / 23(30)$ & $5 / 6(83)$ & $1 / 1(100)$ & 0.004 \\
\hline
\end{tabular}

$(P>0.2)$ and age $(P=0.1)$ did not significantly moderate the effects of disappointment/death life events on outcome.

To test for independence of clinical and life-event predictors of end-point depression, a multiple regression was performed with CDRS-R, obsessive-compulsive disorder, suicidality, presence/ absence of at least one disappointing life event (the most strong life event predictor) and the potential confounders of site, treatment group, age and gender as covariates. Table 5 demonstrates that obsessive-compulsive disorder, severity and having at least one disappointing life event were significantly independently associated with (and there was a trend for suicidality to be associated with) risk of end-point depression.

Which parent(s) the adolescent lived with had no effect on having depression at end-point (end-point rate: living with both parents, 21/72 (29\%); mother only, 17/88 (19\%); father only, 2/ $6(33 \%)$, Fisher's exact test $P=0.3)$. Household income (only available for 127 cases) had no effect on end-point depression (mean: end-point depression, $\mathfrak{E 3 3 7 1 9}$ (s.d.=38 825); no depression, $£ 32138$ (s.d. $=36491$ ), $t=0.2, P=0.8$ ).

Of 146 adolescents living with their mother for whom data was available, mother's current mental health at presentation was not significantly associated with adolescent end-point depression (mean GHQ: depression, 34.9 (s.d.=17.4); no depression, 34.5 (s.d.=18.0), $t=0.3, P=0.8$ ). Of 55 adolescents living with their father for whom data was available, father's mental health was not quite significantly associated with adolescent endpoint depression (mean GHQ: depression, 28.2 (s.d.=16.2); no depression, 20.2 (s.d.=10.0), $t=1.8, P=0.07$ ).

\section{Receiver operating curve analysis}

Variables that were significantly associated with end-point depression on univariate analyses (severity (CDRS-R), number of depressive symptoms, number of comorbid psychiatric diagnoses, suicidality, guilt, hopelessness, obsessive-compulsive disorder, anxiety disorder, simple phobia, total number of unpleasant life events, number of disappointing life events and number of loss by death life events) together with site and treatment group were entered into the exploratory ROC4 analysis. Results are shown in Fig. 1. The most efficient predictor of outcome was overall severity of depression (as measured by CDRS-R), at quite a high cut-off of $\geqslant 71$, equivalent to a T-score of 83 (greater than 3 standard deviations higher than the population mean). Within those with mild to moderate severity $(\leqslant 70)$, presence of at least one disappointing life event over the course of the study was the most significant predictor.

A secondary ROC analysis not including life events demonstrated obsessive-compulsive disorder to be the most efficient predictor of end-point depression in the group with CDRS-R $<71$ (risk of end-point depression if CDRS-R $\leqslant 70$ : obsessive-compulsive disorder absent, 13.4\%; obsessive-compulsive disorder present, $\left.33.3 \% ; \kappa(0.5,0)=0.220, \chi^{2}=7.6, P<0.01\right)$.

\section{Comparison of changes in clinical variables between groups}

Although severity of depression (CDRS-R), obsessive-compulsive disorder and suicidality increased risk of end-point depression, Table 6 demonstrates that all of these variables significantly improved over 28 weeks in both groups (those with and without end-point depression). Analysis of covariance demonstrated that the fall in CDRS-R was greater in those without than with endpoint depression $(F=140$, d.f. $=1,172, P<0.001)$.

\section{Discussion}

At 28 weeks follow-up, $23 \%$ of the individuals with unipolar major depression remained resistant to the effects of a comprehensive treatment programme delivered in CAMHS out-patients by

Table 5 Multiple logistic regression demonstrating significant clinical and environmental predictors for end-point depression ${ }^{\mathrm{a}}$

\begin{tabular}{|c|c|c|c|}
\hline & Corrected OR $(95 \% \mathrm{Cl})$ & Wald & $P$ \\
\hline Children's Depression Rating Scale - Revised & $1.06(1.01-1.12)$ & 4.90 & 0.049 \\
\hline$\geqslant 1$ disappointing life event & $3.27(1.14-9.37)$ & 4.84 & 0.028 \\
\hline Obsessive-compulsive disorder & $2.37(1.01-5.58)$ & 3.89 & 0.049 \\
\hline Suicidality & $2.74(0.99-7.59)$ & 3.73 & 0.054 \\
\hline Treatment group & $1.03(0.46-2.35)$ & 0.01 & 0.9 \\
\hline Treatment site & $0.02(0.41-2.55)$ & 0.00 & 1.0 \\
\hline Gender & $0.66(0.25-1.71)$ & 0.74 & 0.4 \\
\hline Age & $0.90(0.65-1.26)$ & 0.37 & 0.5 \\
\hline
\end{tabular}




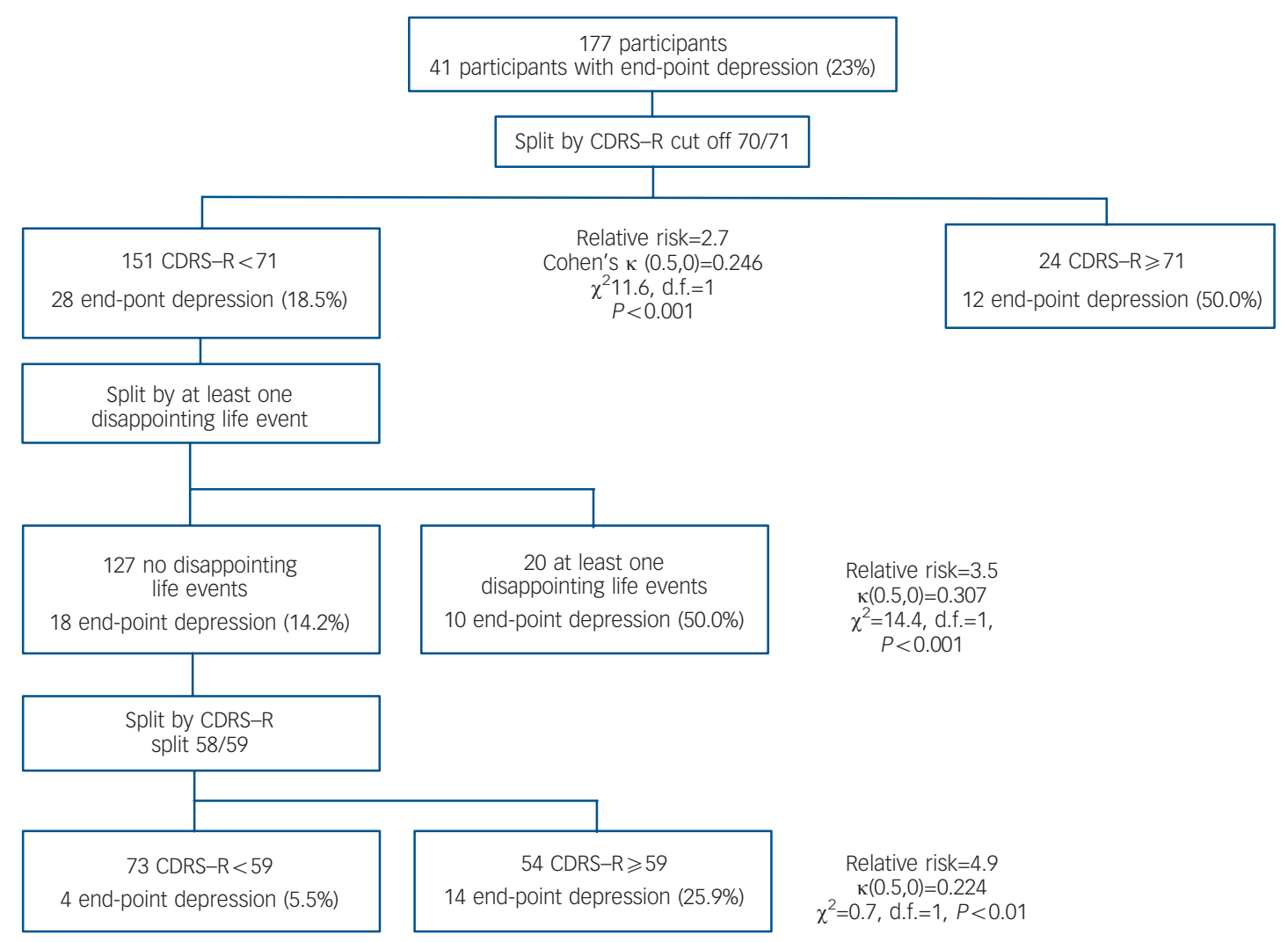

Fig. 1 Results of a receiver operating curve analysis (ROC4) analysis, showing best predictors of end-point depression. CDRS-R, Children's Depression Rating Scale - Revised.

trained and supervised mental health staff. This CAMHS UK sample was considerably more ill and treatment-resistant than has been reported in randomised controlled trials (RCTs) in this age range to date. The results of this analysis are likely to be more applicable than overseas studies to the adolescent population with depression seen in British CAMHS clinics.

Whether an adolescent with moderate to severe unipolar major depression in this sample had end-point disorder 28 weeks later was predicted by the additive effects of severity (as measured by any of the instruments used) and number of comorbid psychiatric disorders. This replicates the findings of previous studies. ${ }^{3,5,6}$ Detailed analysis showed a specific prediction for the additive effects of higher suicidality (thoughts and/or actions), obsessivecompulsive disorder and severity on the liability for end-point depression at 28 weeks. The finding for obsessive-compulsive disorder as a predictor has not previously been reported by other RCTs. These findings are consistent with naturalistic longitudinal findings from other out-patient samples of adolescents with depression that have demonstrated that suicidality ${ }^{3}$ and obsessive-compulsive disorder ${ }^{5}$ are associated with a lower treatment response and/or higher recurrence risk of depression, independently of severity. Interestingly, we could not replicate prior findings that duration of episode nor melancholic features ${ }^{3}$ influence outcome.

Aspects of the adolescent's social environment predicted outcome independently of age and gender of the sample. The number of adverse life events over the course of the study increased the chance of end-point depression. More specifically, this study replicated prior findings ${ }^{11,12,27}$ that it is the disappointment and loss by death categories of life events that predict persistence of an episode of depression, independently of severity.

The exploratory ROC4 analysis suggested that the most efficient predictor of depression at 28 weeks was overall severity of depressive symptoms, which is also the most reliably found predictor in the literature. Within the moderate severity group (CDRS-R <71), the occurrence of at least one unpleasant disappointing life event was an additional predictor of end-point depression. The analysis suggests that in the small group of

\begin{tabular}{|c|c|c|c|c|c|c|}
\hline \multirow[b]{3}{*}{ Baseline clinical variable } & \multicolumn{6}{|c|}{ Depression status at 28 weeks } \\
\hline & \multicolumn{3}{|c|}{ Without depression } & \multicolumn{3}{|c|}{ With depression } \\
\hline & 0 week & 28 week & 0 v. 28 week & 0 week & 28 week & 0 v. 28 week \\
\hline $\begin{array}{l}\text { Children's Depression Rating } \\
\text { Scale - Revised, mean (s.d.) }\end{array}$ & $58.5(8.8)$ & $29.5(9.4)$ & $\begin{array}{c}t=76^{\mathrm{a}} \\
P<0.001\end{array}$ & $65.5(9.7)$ & $56.1(9.8)$ & $\begin{aligned} t & =42^{\mathrm{a}} \\
P & <0.001\end{aligned}$ \\
\hline Suicidality, $n / N(\%)$ & $71 / 136(52)$ & 4/136 (3) & $P<0.001^{\mathrm{b}}$ & $34 / 41$ (83) & $21 / 41(50)$ & $P=0.002^{\mathrm{b}}$ \\
\hline Obsessive-compulsive disorder, $n / N(\%)$ & $30 / 136(22)$ & $2 / 136(1)$ & $P<0.001^{b}$ & $19 / 39(49)$ & $10 / 41(24)$ & $P=0.013^{b}$ \\
\hline
\end{tabular}


adolescents with depression with the lowest severity scores $(<59)$ and no disappointing life events during treatment, there is likely to be a greater than $95 \%$ chance of remission by 28 weeks. Whether this group could be considered at high probability for spontaneous remission is not known. Secondary ROC4 analysis demonstrated that obsessive-compulsive disorder is the most efficient clinical predictor of outcome in those with moderately severe depression (CDRS-R $<71$ ), again demonstrating the importance of this comorbid syndrome. Due to the nature of the ROC4 analysis (splitting the sample into progressively smaller groups) there was not enough power to detect significant effects of other clinical predictors. Overall, however, the ROC analysis suggests that using this pattern of clinical and social predictors alone will not produce either a highly specific or sensitive model for determining the success of these treatments. Genetic and other biological tests may increase predictive sensitivity.

Although high CDRS-R, suicidality and obsessive-compulsive disorder all increased the risk of end-point depression, there were significant improvements in all of these variables in both those with and without end-point depression. The reduction in depression severity was significantly greater in the group without depression at the end. Therefore, these factors genuinely predict differential response to treatment rather than it being the case that one group had higher baseline depression and so were less likely to improve to below the threshold for diagnosis, despite identical degrees of improvement. It is of particular note that suicidality decreased in both groups, despite the fact that all participants were assigned an SSRI antidepressant. Selective serotonin reuptake inhibitors have been demonstrated to slightly increase the risk for suicidal thoughts and acts compared with placebo in RCTs of adolescents with depression. ${ }^{28}$ This has led to advice from European and American regulatory agencies to use SSRIs with great caution in this age group. The findings from this study reflect findings from both the parent ADAPT study and the American Treatment for Adolescents with Depression Study (TADS) that levels of suicidality fell across all treatment groups in both trials. A further conclusion from this study is that treatment with antidepressants is more likely to reduce than increase suicidality, even among those individuals with a depression that is less likely to respond to treatment; however, it must not be forgotten that there are a small number of individuals who will become more suicidal when given an SSRI. Also, the lack of a placebo arm does not allow us to answer whether there would have been a greater fall in suicidality if an SSRI was not given.

\section{Limitations}

This study was initially planned to compare two different treatments. Therefore, there were no primary hypotheses about risk factors for end-point depression. Multiple statistical testing was carried out, introducing the possible risk of type 1 errors. This report should therefore be seen as hypothesis-generating rather than hypotheses-proving.

The study did not systematically collect data on remission throughout the 28 weeks, so it is not possible to distinguish adolescents who continued to have depression throughout the study and those who went into remission then relapsed. It is possible that different variables predict persistence of the primary episode and a pattern of remission/relapse. In addition, the follow-up period was relatively short and a longer period (of at least a year) would more reliably show which risk factors would predict relapse. Life event data were collected retrospectively, and it is possible that those with depression at the time of final assessment were more likely to recall adverse life events than those in remission, rather than the life events themselves being responsible for the persistent depression.

Many potential participants $(29 \%$ of those identified as depressed in initial clinical assessments) were excluded from the study because they were unsuitable or unwilling to take part in a treatment study, in particular unwillingness to take antidepressants as a first-line treatment. In addition, 34 participants did not enter the study because they went into partial remission during a brief initial intervention. Therefore, the sample may not be truly representative of the whole population of adolescents attending British child and adolescent mental health out-patient clinics. Instead, the sample is more representative of adolescents with non-transient depression severe enough to consider antidepressants. As most participants were on antidepressants, this study only tells us about which risk factors predict end-point depression in the presence of antidepressant treatment, rather than in a naturalistic sample. A different set of risk factors may predict non-response to psychological treatment alone.

None the less, the controlling of treatment, with high rates of staying on antidepressants and the finding of no additional effect of CBT, made it more likely that the baseline clinical factors rather than any treatment effects were responsible for the differences in outcome.

\section{Clinical implications}

From the clinical perspective, CAMHS staff should include in their assessments of adolescents with depression a comprehensive understanding of the level of severity, degree of suicidality and the presence of multiple comorbidities, especially obsessive-compulsive disorder, at first assessment. Monitoring and addressing the social environment during treatment would seem prudent, as further undesirable life events (involving friends as well as family) may reduce treatment response, even in those with non-severe episodes, low suicidality and no obsessive-compulsive disorder. Levels of suicidality are more likely to reduce than increase in adolescents with depression after treatment with an SSRI and specialist psychosocial care, even among those who are more likely to continue to have depression after treatment (as predicted by the above variables).

\footnotetext{
Paul Wilkinson, MRCPsych, MD, Developmental Psychiatry Section, University of Cambridge, Bernadka Dubicka, MRCPsych, Department of Child Psychiatry, University of Manchester, and the Junction Adolescent Unit, Scotforth, Lancaster Raphael Kelvin, MRCPsych, Developmental Psychiatry Section, University of Cambridge; Chris Roberts, PhD, Biostatistics Group, School of Epidemiology \& Health Sciences, University of Manchester; Ian Goodyer, FRCPsych, MD, Developmental Psychiatry Section, University of Cambridge, UK

Correspondence: Paul Wilkinson, Developmental Psychiatry Section, University of Cambridge, Douglas House, 18b Trumpington Road, Cambridge CB2 8AH, UK. Email: pow12@cam.ac.uk

First received 14 Mar 2008, final revision 19 Aug 2008, accepted 23 Sep 2008
}

\section{Funding}

The ADAPT study was funded by the NHS Health Technology Assessment Programme, Central Manchester and Manchester Children's University Hospitals NHS Trust and the Cambridge and Peterborough Mental Health Partnership Trust.

\section{Acknowledgements}

We thank the other members of the ADAPT team: Sarah Byford, Barbara Barrett, Alison Leech, Claire Harris, Siobhan Breen, Justine Rothwell and Lydia White. We in particular wish Leech, Claire Harris, Siobhan Breen, Justine Rothwell and Lydia White. We in particular wish
to thank the late Professor Richard Harrington, inspiration behind and joint principal investigator of the ADAPT study. 


\section{References}

1 Dunn V, Goodyer IM. Longitudinal investigation into childhood- and adolescence-onset depression: psychiatric outcome in early adulthood. Br J Psychiatry 2006; 188: 216-22.

2 Brent DA, Kolko DJ, Allan MJ, Brown RV. Suicidality in affectively disordered adolescent inpatients. J Am Acad Child Adolesc Psychiatry 1990; 29: 586-93.

3 Curry J, Rohde P, Simons A, Silva S, Vitiello B, Kratochvil C, et al. Predictors and moderators of acute outcome in the Treatment for Adolescents with Depression Study (TADS). J Am Acad Child Adolesc Psychiatry 2006; 45: 1427-39.

4 Lewinsohn PM, Clarke GN, Seeley JR, Rohde P. Major depression in community adolescents: age at onset, episode duration, and time to recurrence. J Am Acad Child Adolesc Psychiatry 1994; 33: 809-18.

5 Goodyer IM, Herbert J, Secher SM, Pearson J. Short-term outcome of major depression: I. Comorbidity and severity at presentation as predictors of persistent disorder. J Am Acad Child Adolesc Psychiatry 1997; 36: 179-87.

6 McCauley E, Myers K, Mitchell J, Calderon R, Schloredt K, Treder R. Depression in young people: initial presentation and clinical course. J Am Acad Child Adolesc Psychiatry 1993; 32: 714-22.

7 Brent DA, Kolko DJ, Birmaher B, Baugher M, Bridge J, Roth C, et al. Predictors of treatment efficacy in a clinical trial of three psychosocial treatments for adolescent depression. J Am Acad Child Adolesc Psychiatry 1998; 37: 906-14.

8 Kovacs M, Obrosky DS, Gatsonis C, Richards C. First-episode major depressive and dysthymic disorder in childhood: clinical and sociodemographic factors in recovery. J Am Acad Child Adolesc Psychiatry 1997; 36: 777-84.

9 Fombonne E, Wostear G, Cooper V, Harrington R, Rutter M. The Maudsley long-term follow-up of child and adolescent depression. 1. Psychiatric outcomes in adulthood. Br J Psychiatry 2001; 179: 210-7.

10 Goodyer IM, Herbert J, Tamplin A, Secher SM, Pearson J. Short-term outcome of major depression: II. Life events, family dysfunction, and friendship difficulties as predictors of persistent disorder. J Am Acad Child Adolesc Psychiatry 1997; 36: 474-80.

11 Goodyer I, Germany E, Gowrusankur J, Altham P. Social influences on the course of anxious and depressive disorders in school-age children. Br J Psychiatry 1991; 158: 676-84.

12 Goodyer IM, Herbert J, Altham PM. Adrenal steroid secretion and major depression in 8- to 16-year-olds, III. Influence of cortisol/DHEA ratio at presentation on subsequent rates of disappointing life events and persistent major depression. Psychol Med 1998; 28: 265-73.
13 Goodyer I, Dubicka B, Wilkinson P, Kelvin R, Roberts C, Byford S, et al. Selective serotonin reuptake inhibitors (SSRIS) and routine specialist care with and without cognitive behaviour therapy in adolescents with major depression: randomised controlled trial. BMJ 2007; 335: 142.

14 American Psychiatric Association. Diagnostic and Statistical Manual of Mental Disorder (4th edn) (DSM-IV). APA, 1994

15 Kaufman J, Birmaher B, Brent D, Rao U, Flynn C, Moreci P, et al. Schedule for Affective Disorders and Schizophrenia for School-Age Children-Present and Lifetime Version (K-SADS-PL): initial reliability and validity data. J Am Acad Child Adolesc Psychiatry 1997; 36: 980-8.

16 Costello EJ, Angold A. Scales to assess child and adolescent depression: checklists, screens and nets. J Am Acad Child Adolesc Psychiatry 1988; 27 726-37

17 Poznanski EO, Grossman JA, Buchsbaum Y, Banegas M, Freeman L, Gibbons R. Preliminary studies of the reliability and validity of the children's depression rating scale. J Am Acad Child Adolesc Psychiatry 1984; 23: 191-7.

18 Gowers SG, Harrington RC, Whitton A, Lelliott P, Beevor A, Wing J, et al. Brief scale for measuring the outcomes of emotional and behavioural disorders in children. Health of the Nation Outcome Scales for children and Adolescents (HoNOSCA). Br J Psychiatry 1999; 174: 413-6.

19 Shaffer D, Gould MS, Brasic J, Ambrosini P, Fisher P, Bird H, et al. A children's global assessment scale (CGAS). Arch Gen Psychiatry 1983; 40: 1228-31.

20 Goldberg DP. General Health Questionnaire-28. The Institute of Psychiatry, 1981.

21 Brown GW, Bifulco A, Harris TO. Life events, vulnerability and onset of depression: some refinements. Br J Psychiatry 1987; 150: 30-42.

22 Goodyer I, Kolvin I, Gatzanis S. Recent undesirable life events and psychiatric disorder in childhood and adolescence. Br J Psychiatry 1985; 147: 517-23.

23 Goodyer IM, Herbert J, Tamplin A, Altham PME. Recent life events, cortisol, dehydroepiandrosterone and the onset of major depression in high-risk adolescents. Br J Psychiatry 2000; 177: 499-504.

24 Monroe SM, Rohde P, Seeley JR, Lewinsohn PM. Life events and depression in adolescence: relationship loss as a prospective risk factor for first onset of major depressive disorder. J Abnorm Psychol 1999; 108: 606-14.

25 Kraemer HC. Evaluating Medical Tests: Objective and Quantitative Guidelines. Sage Publications, 1992

26 Kraemer $\mathrm{H}$. Signal detection methods and predictions of treatment response (ROC4 program download) 2004. (http://mirecc.stanford.edu/streams.html).

27 Eley TC, Stevenson J. Specific life events and chronic experiences differentially associated with depression and anxiety in young twins. J Abnorm Child Psychol 2000; 28: 383-94.

28 Hammad TA, Laughren T, Racoosin J. Suicidality in pediatric patients treated with antidepressant drugs. Arch Gen Psychiatry 2006; 63: 332-9. 